\title{
ASYMPTOTICALLY HOLOMORPHIC FAMILIES OF SYMPLECTIC SUBMANIFOLDS
}

\author{
DENIS AUROUX
}

\begin{abstract}
We construct a wide range of symplectic submanifolds in a compact symplectic manifold as the zero sets of asymptotically holomorphic sections of vector bundles obtained by tensoring an arbitrary vector bundle by large powers of the complex line bundle whose first Chern class is the symplectic form. We also show that, asymptotically, all sequences of submanifolds constructed from a given vector bundle are isotopic. Furthermore, we prove a result analogous to the Lefschetz hyperplane theorem for the constructed submanifolds.
\end{abstract}

\section{INTRODUCTION}

In a recent paper [1], Donaldson has exhibited an elementary construction of symplectic submanifolds of codimension 2 in a compact symplectic manifold, where the submanifolds are seen as the zero sets of asymptotically holomorphic sections of well-chosen line bundles. In this paper, we extend this construction to higher rank bundles as well as one-parameter families, and obtain as a consequence an important isotopy result.

In all the following, $(X, \omega)$ will be a compact symplectic manifold of dimension $2 n$, such that the cohomology class $\left[\frac{\omega}{2 \pi}\right]$ is integral. A compatible almost-complex structure $J$ and the corresponding riemannian metric $g$ are fixed. Let $L$ be the complex line bundle on $X$ whose first Chern class is $c_{1}(L)=\left[\frac{\omega}{2 \pi}\right]$. Fix a hermitian structure on $L$, and let $\nabla^{L}$ be a hermitian connection on $L$ whose curvature 2 -form is equal to $-i \omega$ (it is clear that such a connection always exists).

We will consider families of sections of bundles of the form $E \otimes L^{k}$ on $X$, defined for all large values of an integer parameter $k$, where $E$ is any hermitian vector bundle over $X$. The connection $\nabla^{L}$ induces a connection of curvature $-i k \omega$ on $L^{k}$, and together with any given hermitian connection $\nabla^{E}$ on $E$ this yields a hermitian connection on $E \otimes L^{k}$ for any $k$. We are interested in sections which satisfy the following two properties :

Definition 1. A sequence of sections $s_{k}$ of $E \otimes L^{k}$ (for large $k$ ) is said to be asymptotically holomorphic with respect to the given connections and almost-complex structure if the following bounds hold:

$$
\begin{array}{lll}
\left|s_{k}\right|=O(1), & \left|\nabla s_{k}\right|=O\left(k^{1 / 2}\right), & \left|\bar{\partial} s_{k}\right|=O(1), \\
& \left|\nabla \nabla s_{k}\right|=O(k), & \left|\nabla \bar{\partial} s_{k}\right|=O\left(k^{1 / 2}\right) .
\end{array}
$$


Since $X$ is compact, up to a change by a constant factor in the estimates, the notion of asymptotic holomorphicity does not actually depend on the chosen hermitian structures and on the chosen connection $\nabla^{E}$. On the contrary, the connection $\nabla^{L}$ is essentially determined by the symplectic form $\omega$, and the positivity property of its curvature is the fundamental ingredient that makes the construction possible.

Definition 2. A section s of a vector bundle $E \otimes L^{k}$ is said to be $\eta$-transverse to 0 if whenever $|s(x)|<\eta$, the covariant derivative $\nabla s(x): T_{x} X \rightarrow$ $\left(E \otimes L^{k}\right)_{x}$ is surjective and admits a right inverse whose norm is smaller than $\eta^{-1} \cdot k^{-1 / 2}$. A family of sections is transverse to 0 if there exists an $\eta>0$ such that $\eta$-transversality to 0 holds for all large values of $k$.

In the case of line bundles, $\eta$-transversality to 0 simply means that the covariant derivative of the section is larger than $\eta k^{1 / 2}$ wherever the section is smaller than $\eta$. Also note that transversality to 0 is an open property : if $s$ is $\eta$-transverse to 0 , then any section $\sigma$ such that $|s-\sigma|<\epsilon$ and $|\nabla s-\nabla \sigma|<k^{1 / 2} \epsilon$ is automatically $(\eta-\epsilon)$-transverse to 0 . The following holds clearly, independently of the choice of the connections on the vector bundles :

Proposition 1. Let $s_{k}$ be sections of the vector bundles $E \otimes L^{k}$ which are simultaneously asymptotically holomorphic and transverse to 0 . Then for all large enough $k$, the zero sets $W_{k}$ of $s_{k}$ are embedded symplectic submanifolds in $X$. Furthermore, the submanifolds $W_{k}$ are asymptotically J-holomorphic, i.e. $J\left(T W_{k}\right)$ is within $O\left(k^{-1 / 2}\right)$ of $T W_{k}$.

The result obtained by Donaldson [1] can be expressed as follows :

Theorem 1. For all large $k$ there exist sections of the line bundles $L^{k}$ which are transverse to 0 and asymptotically holomorphic (with respect to connections of curvature $-i k \omega$ on $L^{k}$ ).

Our main result is the following (the extension to almost-complex structures that depend on $t$ was suggested by the referee) :

Theorem 2. Let $E$ be a complex vector bundle of rank $r$ over $X$, and let a parameter space $T$ be either $\{0\}$ or $[0,1]$. Let $\left(J_{t}\right)_{t \in T}$ be a family of almostcomplex structures on $X$ compatible with $\omega$. Fix a constant $\epsilon>0$, and let $\left(s_{t, k}\right)_{t \in T, k \geq K}$ be a sequence of families of asymptotically $J_{t}$-holomorphic sections of $E \otimes L^{k}$ defined for all large $k$, such that the sections $s_{t, k}$ and their derivatives depend continuously on $t$.

Then there exist constants $\tilde{K} \geq K$ and $\eta>0$ (depending only on $\epsilon$, the geometry of $X$ and the bounds on the derivatives of $s_{t, k}$ ), and a sequence $\left(\sigma_{t, k}\right)_{t \in T, k \geq \tilde{K}}$ of families of asymptotically $J_{t}$-holomorphic sections of $E \otimes L^{k}$ defined for all $k \geq \tilde{K}$, such that

(a) the sections $\sigma_{t, k}$ and their derivatives depend continuously on $t$,

(b) for all $t \in T,\left|\sigma_{t, k}-s_{t, k}\right|<\epsilon$ and $\left|\nabla \sigma_{t, k}-\nabla s_{t, k}\right|<k^{1 / 2} \epsilon$,

(c) for all $t \in T, \sigma_{t, k}$ is $\eta$-transverse to 0 . 
Note that, since we allow the almost-complex structure on $X$ to depend on $t$, great care must be taken as to the choice of the metric on $X$ used for the estimates on derivatives. The most reasonable choice, and the one which will be made in the proof, is to always use the same metric, independently of $t$ (so, there is no relation between $g, \omega$ and $J_{t}$ ). However, it is clear from the statement of the theorem that, since the spaces $X$ and $T$ are compact, any change in the choice of metric can be absorbed by simply changing the constants $\tilde{K}$ and $\eta$, and so the result holds in all generality.

Theorem 2 has many consequences. Among them, we mention the following extension of Donaldson's result to higher rank bundles :

Corollary 1. For any complex vector bundle $E$ over $X$ and for all large $k$, there exist asymptotically holomorphic sections of $E \otimes L^{k}$ which are transverse to 0 , and thus whose zero sets are embedded symplectic submanifolds in $X$. Furthermore given a sequence of asymptotically holomorphic sections of $E \otimes L^{k}$ and a constant $\epsilon>0$, we can require that the transverse sections lie within $\epsilon$ in $C^{0}$ sense (and $k^{1 / 2} \epsilon$ in $C^{1}$ sense) of the given sections.

Therefore, the homology classes that one can realize by this construction include all classes whose Poincaré dual is of the form $\left[\frac{k \omega}{2 \pi}\right]^{r}+c_{1} \cdot\left[\frac{k \omega}{2 \pi}\right]^{r-1}+$ $\ldots+c_{r}$, with $c_{1}, \ldots, c_{r}$ the Chern classes of any complex vector bundle and $k$ any sufficiently large integer.

An important result that one can obtain on the sequences of submanifolds constructed using Corollary 1 is the following isotopy result derived from the case where $T=[0,1]$ in Theorem 2 and which had been conjectured by Donaldson in the case of line bundles :

Corollary 2. Let $E$ be any complex vector bundle over $X$, and let $s_{0, k}$ and $s_{1, k}$ be two sequences of sections of $E \otimes L^{k}$. Assume that these sections are asymptotically holomorphic with respect to almost-complex structures $J_{0}$ and $J_{1}$ respectively, and that they are $\epsilon$-transverse to 0 . Then for all large $k$ the zero sets of $s_{0, k}$ and $s_{1, k}$ are isotopic through asymptotically holomorphic symplectic submanifolds. Moreover, this isotopy can be realized through symplectomorphisms of $X$.

This result follows from Theorem 2 by defining sections $s_{t, k}$ and almostcomplex structures $J_{t}$ that interpolate between $\left(s_{0, k}, J_{0}\right)$ and $\left(s_{1, k}, J_{1}\right)$ in the following way : for $t \in\left[0, \frac{1}{3}\right]$, let $s_{t, k}=(1-3 t) s_{0, k}$ and $J_{t}=J_{0}$; for $t \in\left[\frac{1}{3}, \frac{2}{3}\right]$, let $s_{t, k}=0$ and take $J_{t}$ to be a path of compatible almost-complex structures from $J_{0}$ to $J_{1}$ (this is possible since the space of compatible almost-complex structures is connected); and for $t \in\left[\frac{2}{3}, 1\right]$, let $s_{t, k}=(3 t-2) s_{1, k}$ and $J_{t}=J_{1}$. One can then apply Theorem 2 and obtain for all large $k$ and for all $t \in[0,1]$ sections $\sigma_{t, k}$ that differ from $s_{t, k}$ by at most $\epsilon / 2$ and are $\eta$-transverse to 0 for some $\eta$. Since transversality to 0 is an open property, the submanifolds cut out by $\sigma_{0, k}$ and $\sigma_{1, k}$ are clearly isotopic to those cut out by $s_{0, k}$ and $s_{1, k}$. Moreover, the family $\sigma_{t, k}$ gives an isotopy between the zero sets of $\sigma_{0, k}$ and $\sigma_{1, k}$. So the constructed submanifolds are isotopic. The proof that this 
isotopy can be realized through symplectomorphisms of $X$ will be given in Section 4.

As a first step in the characterization of the topology of the constructed submanifolds, we also prove the following statement, extending the result obtained by Donaldson in the case of the line bundles $L^{k}$ :

Proposition 2. Let $E$ be a vector bundle of rank $r$ over $X$, and let $W_{k}$ be a sequence of symplectic submanifolds of $X$ constructed as the zero sets of asymptotically holomorphic sections $s_{k}$ of $E \otimes L^{k}$ which are transverse to 0 , for all large $k$. Then when $k$ is sufficiently large, the inclusion $i: W_{k} \rightarrow$ $X$ induces an isomorphism on homotopy groups $\pi_{p}$ for $p<n-r$, and $a$ surjection on $\pi_{n-r}$. The same property also holds for homology groups.

Section 2 contains the statement and proof of the local result on which the whole construction relies. Section 3 deals with the proof of a semi-global statement, using a globalization process to obtain results on large subsets of $X$ from the local picture. The proofs of Theorem 2 and Corollary 2 are then completed in Section 4. Section 5 contains miscellaneous results on the topology and geometry of the obtained submanifolds, including Proposition 2 .

Acknowledgments. The author wishes to thank Professor Mikhael Gromov (IHES) for valuable suggestions and guidance throughout the elaboration of this paper, and Professor Jean-Pierre Bourguignon (Ecole Polytechnique) for his support.

\section{The LOCAL RESUlT}

The proof of Theorem 2 relies on a local transversality result for approximatively holomorphic functions, which we state and prove immediately.

Proposition 3. There exists an integer $p$ depending only on the dimension $n$, with the following property : let $\delta$ be a constant with $0<\delta<\frac{1}{2}$, and let $\sigma=\delta \cdot \log \left(\delta^{-1}\right)^{-p}$. Let $\left(f_{t}\right)_{t \in T}$ be a family of complex-valued functions over the ball $B^{+}$of radius $\frac{11}{10}$ in $\mathbb{C}^{n}$, depending continuously on the parameter $t \in T$ and satisfying for all $t$ the following bounds over $B^{+}$:

$$
\left|f_{t}\right| \leq 1, \quad\left|\bar{\partial} f_{t}\right| \leq \sigma, \quad\left|\nabla \bar{\partial} f_{t}\right| \leq \sigma .
$$

Then there exists a family of complex numbers $w_{t} \in \mathbb{C}$, depending continuously on $t$, such that for all $t \in T,\left|w_{t}\right| \leq \delta$, and $f_{t}-w_{t}$ has a first derivative larger than $\sigma$ at any point of the interior ball $B$ of radius 1 where its norm is smaller than $\sigma$.

Proposition 3 extends a similar result proved in detail in [1], which corresponds to the case where $T=\{0\}$. The proof of Proposition 3 is based on the same ideas as Donaldson's proof, which is in turn based on considerations from real algebraic geometry following the method of Yomdin [7][3], with the only difference that we must get everything to depend continuously 
on $t$. Note that this statement is false for more general parameter spaces $T$ than $\{0\}$ and $[0,1]$, since for example when $T$ is the unit disc in $\mathbb{C}$ and $f_{t}(z)=t$, one looks for a continuous map $t \mapsto w_{t}$ of the disc to itself without a fixed point, in contradiction with Brouwer's theorem.

The idea is to deal with polynomial functions $g_{t}$ approximating $f_{t}$, for which a general result on the complexity of real semi-algebraic sets gives constraints on the near-critical levels. This part of the proof is similar to that given in [1], so we skip the details. To obtain polynomial functions, we approximate $f_{t}$ first by a continuous family of holomorphic functions $\tilde{f}_{t}$ differing from $f_{t}$ by at most a fixed multiple of $\sigma$ in $C^{1}$ sense, using that $\bar{\partial} f_{t}$ is small. The polynomials $g_{t}$ are then obtained by truncating the Taylor series expansion of $\tilde{f}_{t}$ to a given degree. It can be shown that by this method one can obtain polynomial functions $g_{t}$ of degree $d$ less than a constant times $\log \left(\sigma^{-1}\right)$, such that $g_{t}$ differs from $f_{t}$ by at most $c . \sigma$ in $C^{1}$ sense, where $c$ is a fixed constant (see [1]). This approximation process does not hold on the whole ball where $f_{t}$ is defined, which is why we needed $f_{t}$ to be defined on $B^{+}$to get a result over the slightly smaller ball $B$ (see Lemmas 27 and 28 of [1]).

For a given complex-valued function $h$ over $B$, call $Y_{h, \epsilon}$ the set of all points in $B$ where the derivative of $h$ has norm less than $\epsilon$, and call $Z_{h, \epsilon}$ the $\epsilon$-tubular neighborhood of $h\left(Y_{h, \epsilon}\right)$. What we wish to construct is a path $w_{t}$ avoiding by at least $\sigma$ all near-critical levels of $f_{t}$, i.e. consisting of values that lie outside of $Z_{f_{t}, \sigma}$. Since $g_{t}$ is within $c . \sigma$ of $f_{t}$, it is clear that $Z_{f_{t}, \sigma}$ is contained in $Z_{t}=Z_{g_{t},(c+1) \sigma}$. However a general result on the complexity of real semi-algebraic sets yields constraints on the set $Y_{g_{t},(c+1) \sigma}$. The precise statement which one applies to the real polynomial $\left|d g_{t}\right|^{2}$ is the following (Proposition 25 of [1]) :

Lemma 1. Let $F: \mathbb{R}^{m} \rightarrow \mathbb{R}$ be a polynomial function of degree $d$, and let $S(\theta) \subset \mathbb{R}^{m}$ be the subset $S(\theta)=\left\{x \in \mathbb{R}^{m}:|x| \leq 1, F(x) \leq 1+\theta\right\}$. Then for arbitrarily small $\theta>0$ there exist fixed constants $C$ and $\nu$ depending only on the dimension $m$ such that $S(0)$ may be decomposed into pieces $S(0)=S_{1} \cup S_{2} \cdots \cup S_{A}$, where $A \leq C d^{\nu}$, in such a way that any pair of points in the same piece $S_{r}$ can be joined by a path in $S(\theta)$ of length less than $C d^{\nu}$.

So, as described in [1], given any fixed $t$, the set $Y_{g_{t},(c+1) \sigma}$ of near-critical points of the polynomial function $g_{t}$ of degree $d$ can be subdivided into at most $P(d)$ subsets, where $P$ is a fixed polynomial, in such a way that two points lying in the same subset can be joined by a path of length at most $P(d)$ inside $Y_{g_{t}, 2(c+1) \sigma}$. It follows that the image by $g_{t}$ of $Y_{g_{t},(c+1) \sigma}$ is contained in the union of $P(d)$ discs of radius at most $2(c+1) \sigma P(d)$, so that the set $Z_{t}$ of values which we wish to avoid is contained in the union $Z_{t}^{+}$of $P(d)$ discs of radius $\sigma Q(d)$, where $Q=3(c+1) P$ is a fixed polynomial and $d=O\left(\log \sigma^{-1}\right)$. 
If one assumes $\delta$ to be larger than $\sigma Q(d) P(d)^{1 / 2}$, it follows immediately from this constraint on $Z_{t}$ that $Z_{t}$ cannot fill the disc $D$ of all complex numbers of norm at most $\delta$ : this immediately proves the case $T=\{0\}$. However, when $T=[0,1]$, we also need $w_{t}$ to depend continuously on $t$. For this purpose, we show that if $\delta$ is large enough, $D-Z_{t}^{+}$, when decomposed into connected components, splits into several small components and only one large component.

Indeed, given a component $C$ of $D-Z_{t}^{+}$, the simplest situation is that it does not meet the boundary of $D$. Then its boundary is a curve consisting of pieces of the boundaries of the balls making up $Z_{t}^{+}$, so its length is at most $2 \pi P(d) Q(d) \sigma$, and it follows that $C$ has diameter less than $\pi P(d) Q(d) \sigma$. Considering two components $C_{1}$ and $C_{2}$ which meet the boundary of $D$ at points $z_{1}$ and $z_{2}$, we can consider an arc $\gamma$ joining the boundary of $D$ to itself that separates $C_{1}$ from $C_{2}$ and is contained in the boundary of $Z_{t}^{+}$. Assuming that $\delta$ is larger than e.g. $100 P(d) Q(d) \sigma$, since the length of $\gamma$ is at most $2 \pi P(d) Q(d) \sigma$, it must stay close to either $z_{1}$ or $z_{2}$ in order to separate them : $\gamma$ must remain within a distance of at most $10 P(d) Q(d) \sigma$ from one of them. It follows that there exists $i \in\{1,2\}$ such that $C_{i}$ is contained in the ball of radius $10 P(d) Q(d) \sigma$ centered at $z_{i}$. So all components of $D-Z_{t}^{+}$except at most one are contained in balls of radius $R(d) \sigma$, for some fixed polynomial $R$. Furthermore, the number of components of $D-Z_{t}^{+}$is bounded by a value directly related to the number of balls making up $Z_{t}^{+}$, so that, increasing $R$ if necessary, the number of components of $D-Z_{t}^{+}$is also bounded by $R(d)$.

Assuming that $\delta$ is much larger than $R(d)^{3 / 2} \sigma$, the area $\pi \delta^{2}$ of $D$ is much larger than $\pi R(d)^{3} \sigma^{2}$, so that the small components of $D-Z_{t}^{+}$cannot fill it, and there must be a single large component. Getting back to $D-Z_{t}$, which was the set in which we had to choose $w_{t}$, it contains $D-Z_{t}^{+}$and differs from it by at most $Q(d) \sigma$, so that, letting $U(t)$ be the component of $D-Z_{t}$ containing the large component of $D-Z_{t}^{+}$, it is the only large component of $D-Z_{t}$. The component $U(t)$ is characterized by the property that it is the only component of diameter more than $2 R(d) \sigma$ in $D-Z_{t}$.

So the existence of a single large component $U(t)$ in $D-Z_{t}$ is proved upon the assumption that $\delta$ is large enough, namely larger than $\sigma . \Phi(d)$ where $\Phi$ is a given fixed polynomial that can be expressed in terms of $P, Q$ and $R$ (so $\Phi$ depends only on the dimension $n$ ). Since $d$ is bounded by a constant times $\log \sigma^{-1}$, it is not hard to see that there exists an integer $p$ such that, for all $0<\delta<\frac{1}{2}$, the relation $\sigma=\delta \cdot \log \left(\delta^{-1}\right)^{-p}$ implies that $\delta>\sigma . \Phi(d)$. This is the value of $p$ which we choose in the statement of the proposition, thus ensuring that the above statements always hold.

Since $\bigcup_{t}\{t\} \times Z_{t}$ is a closed subset of $T \times D$, the open set $U(t)$ depends semi-continuously on $t$ : let $U^{-}(t, \epsilon)$ be the set of all points of $U(t)$ at distance more than $\epsilon$ from $Z_{t} \cup \partial D$. We claim that, given any $t$ and any small $\epsilon>0$, for all $\tau$ close enough to $t, U(\tau)$ contains $U^{-}(t, \epsilon)$. To see this, we first show for all $\tau$ close to $t, U^{-}(t, \epsilon) \cap Z_{\tau}=\emptyset$. Assuming that such is 
not the case, one can get a sequence of points of $Z_{\tau}$ for $\tau \rightarrow t$ that belong to $U^{-}(t, \epsilon)$. From this sequence one can extract a convergent subsequence, whose limit belongs to $\bar{U}^{-}(t, \epsilon)$ and thus lies outside of $Z_{t}$, in contradiction with the fact that $\bigcup_{t}\{t\} \times Z_{t}$ is closed. So $U^{-}(t, \epsilon) \subset D-Z_{\tau}$ for all $\tau$ close enough to $t$. Making $\epsilon$ smaller if necessary, one may assume that $U^{-}(t, \epsilon)$ is connected, so that for $\tau$ close to $t, U^{-}(t, \epsilon)$ is necessarily contained in the large component of $D-Z_{\tau}$, namely $U(\tau)$.

It follows that $U=\bigcup_{t}\{t\} \times U(t)$ is an open connected subset of $T \times D$, and is thus path-connected. So we get a path $s \mapsto(t(s), w(s))$ joining $(0, w(0))$ to $(1, w(1))$ inside $U$, for any given $w(0)$ and $w(1)$ in $U(0)$ and $U(1)$. We then only have to make sure that $s \mapsto t(s)$ is strictly increasing in order to define $w_{t(s)}=w(s)$.

Getting the $t$ component to increase strictly is in fact quite easy. Indeed, we first get it to be weakly increasing, by considering values $s_{1}<s_{2}$ of the parameter such that $t\left(s_{1}\right)=t\left(s_{2}\right)=t$ and simply replacing the portion of the path between $s_{1}$ and $s_{2}$ by a path joining $w\left(s_{1}\right)$ to $w\left(s_{2}\right)$ in the connected set $U(t)$. Then, we slightly shift the path, using the fact that $U$ is open, to get the $t$ component to increase slightly over the parts where it was constant. Thus we can define $w_{t(s)}=w(s)$ and end the proof of Proposition 3.

\section{The GLOBALIZATION PROCESS}

3.1. Statement of the result. We will now prove a semi-global result using Proposition 3. The globalization process we describe here is based on that used by Donaldson in [1], but a significantly higher amount of work is required because we have to deal with bundles of rank larger than one. The important fact we use is that transversality to 0 is a local and open property.

Theorem 3. Let $U$ be any open subset of $X$, and let $E$ be a complex vector bundle of rank $r \geq 0$ over $U$. Let $\left(J_{t}\right)_{t \in T}$ be a family of almost-complex structures on $X$ compatible with $\omega$. Fix a constant $\epsilon>0$. Let $W_{t, k}$ be a family of symplectic submanifolds in $U$, obtained as the zero sets of asymptotically $J_{t}$-holomorphic sections $w_{t, k}$ of the vector bundles $E \otimes L^{k}$ which are $\eta$-transverse to 0 over $U$ for some $\eta>0$ and depend continuously on $t \in T$ (if the rank is $r=0$, then we simply define $W_{t, k}=U$ ). Finally, let $\left(\sigma_{t, k}\right)$ be a family of asymptotically $J_{t}$-holomorphic sections of $L^{k}$ which depend continuously on $t$. Define $U_{k}^{=}$to be the set of all points of $U$ at distance more than $4 k^{-1 / 3}$ from the boundary of $U$.

Then for some $\tilde{\eta}>0$ and for all large $k$, there exist asymptotically $J_{t^{-}}$ holomorphic sections $\tilde{\sigma}_{t, k}$ of $L^{k}$ over $U$, depending continuously on $t$, and such that

(a) for all $t \in T, \tilde{\sigma}_{t, k}$ is equal to $\sigma_{t, k}$ near the boundary of $U$,

(b) $\left|\tilde{\sigma}_{t, k}-\sigma_{t, k}\right|<\epsilon$ and $\left|\nabla \tilde{\sigma}_{t, k}-\nabla \sigma_{t, k}\right|<k^{1 / 2} \epsilon$ for all $t$,

(c) the sections $\left(w_{t, k}+\tilde{\sigma}_{t, k}\right)$ of $(E \oplus \mathbb{C}) \otimes L^{k}$ are $\tilde{\eta}$-transverse to 0 over $U_{k}^{=}$for all $t$. 
Basically, this result states that the construction of Theorem 2 can be carried out, in the line bundle case, in such a way that the resulting sections are transverse to a given family of symplectic submanifolds.

As remarked in the introduction, the choice of the metric in the statement of the theorem is not obvious. We choose to use always the same metric $g$ on $X$, rather than trying to work directly with the metrics $g_{t}$ induced by $\omega$ and $J_{t}$.

3.2. Local coordinates and sections. The proof of Theorem 3 is based on the existence of highly localized asymptotically holomorphic sections of $L^{k}$ near every point $x \in X$. First, we notice that near any point $x \in X$, we can define local complex Darboux coordinates $\left(z_{i}\right)$, that is to say a symplectomorphism from a neighborhood of $x$ in $(X, \omega)$ to a neighborhood of 0 in $\mathbb{C}^{n}$ with the standard symplectic form. Furthermore it is well-known that, by composing the coordinate map with a (R-linear) symplectic transformation of $\mathbb{C}^{n}$, one can ensure that its differential at $x$ induces a complex linear map from $\left(T_{x} X, J_{t}\right)$ to $\mathbb{C}^{n}$ with its standard complex structure.

Since the almost-complex structure $J_{t}$ is not integrable, the coordinate map cannot be made pseudo-holomorphic on a whole neighborhood of $x$. However, since the manifold $X$ and the parameter space $T$ are compact, the Nijenhuis tensor, which is the obstruction to the integrability of the complex structure $J_{t}$ on $X$, is bounded by a fixed constant, and so are its derivatives. It follows that for a suitable choice of the Darboux coordinates, the coordinate map can be made nearly pseudo-holomorphic around $x$, in the sense that the antiholomorphic part of its differential vanishes at $x$ and grows no faster than a constant times the distance to $x$. Furthermore, it is easy to check that the coordinate map can be chosen to depend continuously on the parameter $t$. So, we have the following lemma :

Lemma 2. Near any point $x \in X$, there exist for all $t \in T$ complex Darboux coordinates depending continuously on $t$, such that the inverse $\psi_{t}$ : $\left(\mathbb{C}^{n}, 0\right) \rightarrow(X, x)$ of the coordinate map is nearly pseudo-holomorphic with respect to the almost-complex structure $J_{t}$ on $X$ and the canonical complex structure on $\mathbb{C}^{n}$. Namely, the map $\psi_{t}$, which trivially satisfies $\left|\nabla \psi_{t}\right|=O(1)$ and $\left|\nabla \nabla \psi_{t}\right|=O(1)$ on a ball of fixed radius around 0 , fails to be pseudoholomorphic by an amount that vanishes at 0 and thus grows no faster than the distance to the origin, i.e. $\left|\bar{\partial} \psi_{t}(z)\right|=O(|z|)$, and $\left|\nabla \bar{\partial} \psi_{t}\right|=O(1)$.

Fix a certain value of the parameter $t \in T$, and consider the hermitian connections with curvature $-i k \omega$ that we have put on $L^{k}$ in the introduction. Near any point $x \in X$, using the local complex Darboux coordinates $\left(z_{i}\right)$ we have just constructed, a suitable choice of a local trivialization of $L^{k}$ leads to the following connection 1-form :

$$
A_{k}=\frac{k}{4} \sum_{j=1}^{n}\left(z_{j} d \bar{z}_{j}-\bar{z}_{j} d z_{j}\right)
$$


(it can be readily checked that $d A_{k}=-i k \omega$ ).

On the standard $\mathbb{C}^{n}$ with connection $A_{k}$, the function defined by $s(z)=$ $\exp \left(-k|z|^{2} / 4\right)$ satisfies the equation $\bar{\partial}_{A_{k}} s=0$ and the bound $\left|\nabla_{A_{k}} s\right|=$ $O\left(k^{1 / 2}\right)$. Multiplying this section by a cut-off function at distance $k^{-1 / 3}$ from the origin whose derivative is small enough, we get a section $\tilde{s}$ with small compact support. Since the coordinate map near $x$ has small antiholomorphic part where $\tilde{s}$ is large, the local sections $\tilde{s} \circ \psi_{t}^{-1}$ of $L^{k}$ defined near $x$ by pullback of $\tilde{s}$ through the coordinate map can be easily checked to be asymptotically holomorphic with respect to $J_{t}$ and $A_{k}$. Thus, for all large $k$ and for any point $x \in X$, extending $\tilde{s} \circ \psi_{t}^{-1}$ by 0 away from $x$, we obtain asymptotically holomorphic sections $s_{t, k, x}$ of $L^{k}$.

Since $T$ is compact, the metrics $g_{t}$ induced on $X$ by $\omega$ and $J_{t}$ differ from the chosen reference metric $g$ by a bounded factor. Therefore, it is clear from the way we constructed the sections $s_{t, k, x}$ that the following statement holds :

Lemma 3. There exist constants $\lambda>0$ and $c_{s}>0$ such that, given any $x \in$ $X$, for all $t \in T$ and large $k$, there exist sections $s_{t, k, x}$ of $L^{k}$ over $X$ with the following properties : the sections $s_{t, k, x}$ are asymptotically $J_{t}$-holomorphic; they depend continuously on $t$; the bound $\left|s_{t, k, x}\right| \geq c_{s}$ holds over the ball of radius $10 k^{-1 / 2}$ around $x$; and finally, $\left|s_{t, k, x}\right| \leq \exp \left(-\lambda k \cdot \operatorname{dist}_{g}(x, .)^{2}\right)$ everywhere on $X$.

3.3. General setup and strategy of proof. In a first step, we wish to obtain sections $\tilde{\sigma}_{t, k}$ of $L^{k}$ over $U$ satisfying all the requirements of Theorem 3 , except that we replace (c) by the weaker condition that the restriction of $\tilde{\sigma}_{t, k}$ to $W_{t, k}$ must be $\hat{\eta}$-transverse to 0 over $W_{t, k} \cap U_{k}^{-}$for some $\hat{\eta}>0$, where $U_{k}^{-}$is the set of all points of $U$ at distance more than $2 k^{-1 / 3}$ from the boundary of $U$. It will be shown later that the transversality to 0 of the restriction to $W_{t, k} \cap U_{k}^{-}$of $\tilde{\sigma}_{t, k}$, together with the bounds on the second derivatives, implies the transversality to 0 of $\left(w_{t, k}+\tilde{\sigma}_{t, k}\right)$ over $U_{k}^{=}$.

To start with, we notice that there exists a constant $c>0$ such that $W_{t, k}$ is trivial at small scale, namely in the ball of radius $10 c . k^{-1 / 2}$ around any point. Indeed, if $r=0$ we just take $c=1$, and otherwise we use the fact that $w_{t, k}$ is $\eta$-transverse to 0 , which implies that at any $x \in W_{t, k}$, $\left|\nabla w_{t, k}(x)\right|>\eta . k^{1 / 2}$. Since $\left|\nabla \nabla w_{t, k}\right|<C_{2} . k$ for some constant $C_{2}$, defining $c=\frac{1}{100} \eta \cdot C_{2}^{-1}$, the derivative $\nabla w_{t, k}$ varies by a factor of at most $\frac{1}{10}$ in the ball $B$ of radius $10 c . k^{-1 / 2}$ around $x$. It follows that $B \cap W_{t, k}$ is diffeomorphic to a ball.

In all the following, we work with a given fixed value of $k$, while keeping in mind that all the constants appearing in the estimates have to be independent of $k$.

For fixed $k$, we consider a finite set of points $x_{i}$ of $U_{k}^{-} \subset U$ such that the balls of radius $c . k^{-1 / 2}$ centered around $x_{i}$ cover $U_{k}^{-}$. A suitable choice of the points ensures that their number is $O\left(k^{n}\right)$. For fixed $D>0$, this set can 
be subdivided into $N$ subsets $S_{j}$ such that the distance between two points in the same subset is at least $D \cdot k^{-1 / 2}$. Furthermore, $N=O\left(D^{2 n}\right)$ can be chosen independent of $k$. The precise value of $D$ (and consequently of $N$ ) will be determined later in the proof.

The idea is to start with the sections $\sigma_{t, k}$ of $L^{k}$ and proceed in steps. Let $\mathcal{N}_{j}$ be the union of all balls of radius $c . k^{-1 / 2}$ around the points of $S_{i}$ for all $i<j$. During the $j$-th step, we start from asymptotically $J_{t^{-}}$ holomorphic sections $\sigma_{t, k, j}$ which satisfy conditions (a) and (b), and such that the restriction of $\sigma_{t, k, j}$ to $W_{t, k}$ is $\eta_{j}$-transverse to 0 over $W_{t, k} \cap \mathcal{N}_{j}$, for some constant $\eta_{j}$ independent of $k$. For the first step, this requirement is void, but we choose $\eta_{0}=\frac{\epsilon}{2}$ in order to obtain a total perturbation smaller than $\epsilon$ at the end of the process. We wish to construct $\sigma_{t, k, j+1}$ from $\sigma_{t, k, j}$ by subtracting small multiples $c_{t, k, x} s_{t, k, x}$ of the sections $s_{t, k, x}$ for $x \in S_{j}$, in such a way that the restrictions of the resulting sections are $\eta_{j+1}$-transverse to 0 , for some small $\eta_{j+1}$, over the intersection of $W_{t, k}$ with all balls of radius $c . k^{-1 / 2}$ around points in $S_{j}$. Furthermore, if the coefficients of the linear combination are chosen much smaller than $\eta_{j}$, transversality to 0 still holds over $W_{t, k} \cap \mathcal{N}_{j}$. Also, since the coefficients $c_{t, k, x}$ are bounded, the resulting sections, which are sums of asymptotically holomorphic sections, remain asymptotically holomorphic. So we need to find, for all $x \in S_{j}$, small coefficients $c_{t, k, x}$ so that $\sigma_{t, k, j}-c_{t, k, x} s_{t, k, x}$ has the desired properties near $x$.

3.4. Obtaining transversality near a point of $S_{j}$. In what follows, $x$ is a given point in $S_{j}$, and $B_{x}$ is the ball of radius $c . k^{-1 / 2}$ around $x$. Let $\Omega$ be the closure of the open subset of $T$ containing all $t$ such that $B_{x} \cap W_{t, k}$ is not empty (when $r=0$, one gets $\Omega=T$ ). When $\Omega$ is empty, it is sufficient to define $c_{t, k, x}=0$ for all $t$. Otherwise, $\Omega=\{0\}$ when $T=\{0\}$, and when $T=[0,1]$ clearly $\Omega$ is a union of disjoint closed intervals. In any case, we choose a component $I$ of $\Omega$, i.e. either a closed interval or a point.

We can then define for all $t \in I$ a point $x_{t}$ belonging to $\overline{B_{x}} \cap W_{t, k}$, in such a way that $x_{t}$ depends continuously on $t$, since $W_{t, k}$ depends continuously on $t$ and always intersects $B_{x}$ in a nice way (when $r=0$ one can simply choose $x_{t}=x$ ). Let $\hat{B}_{t}$ be the ball in $W_{t, k}$ of radius $3 c . k^{-1 / 2}$ (for the metric induced by $g$ ) centered at $x_{t}$. Because of the bounds on the second derivatives of $w_{t, k}$, we know that $\hat{B}_{t}$ contains $B_{x} \cap W_{t, k}$ for all $t \in I$. We now want to define a nearly holomorphic diffeomorphism from a neighborhood of 0 in $\mathbb{C}^{n-r}$ to $\hat{B}_{t}$.

Let $\hat{B}$ be the ball of radius $4 c k^{-1 / 2}$ around 0 in $\mathbb{C}^{n-r}$, and let $\hat{B}^{-}$be the smaller ball of radius $3 c k^{-1 / 2}$ around 0 . We claim the following :

Lemma 4. For all $t \in I$, there exist diffeomorphisms $\theta_{t}$ from $\hat{B}$ to a neighborhood of $x_{t}$ in $W_{t, k}$, depending continuously on $t$, such that $\theta_{t}(0)=x_{t}$ and $\theta_{t}\left(\hat{B}^{-}\right) \supset \hat{B}_{t}$, and satisfying the following estimates over $\hat{B}$ :

$$
\left|\bar{\partial} \theta_{t}\right|=O\left(k^{-1 / 2}\right), \quad\left|\nabla \theta_{t}\right|=O(1), \quad\left|\nabla \bar{\partial} \theta_{t}\right|=O(1), \quad\left|\nabla \nabla \theta_{t}\right|=O\left(k^{1 / 2}\right) .
$$


Proof. Recall that, by Lemma 2, there exist local complex Darboux coordinates on $X$ near $x$ depending continuously on $t$ with the property that the inverse map $\psi_{t}:\left(\mathbb{C}^{n}, 0\right) \rightarrow(X, x)$ satisfies the following bounds at all points at distance $O\left(k^{-1 / 2}\right)$ from $x$ :

$$
\left|\bar{\partial} \psi_{t}\right|=O\left(k^{-1 / 2}\right), \quad\left|\nabla \psi_{t}\right|=O(1), \quad\left|\nabla \bar{\partial} \psi_{t}\right|=O(1), \quad\left|\nabla \nabla \psi_{t}\right|=O(1) .
$$

Let $\mathcal{T}_{t}$ be the kernel of the complex linear map $\partial w_{t, k}\left(x_{t}\right)$ in $T_{x_{t}} X$ : it is within $O\left(k^{-1 / 2}\right)$ of the tangent space to $W_{t, k}$ at $x_{t}$, but $\mathcal{T}_{t}$ is preserved by $J_{t}$. Composing $\psi_{t}$ with a translation and a rotation in $\mathbb{C}^{n}$, one gets maps $\tilde{\psi}_{t}$ satisfying the same requirements as $\psi_{t}$, but with $\tilde{\psi}_{t}(0)=x_{t}$ and such that the differential of $\tilde{\psi}_{t}$ at 0 maps the span of the $n-r$ first coordinates to $\mathcal{T}_{t}$.

Furthermore, $X$ and $T$ are compact, so the metrics $g_{t}$ induced by $\omega$ and $J_{t}$ differ from the reference metric $g$ by at most a fixed constant. It follows that, composing $\tilde{\psi}_{t}$ with a fixed dilation of $\mathbb{C}^{n}$ if necessary, one may also require that the image by $\tilde{\psi}_{t}$ of the ball of radius $3 c k^{-1 / 2}$ around 0 contains the ball of radius $4 c k^{-1 / 2}$ around $x$ for the reference metric $g$. The only price to pay is that $\tilde{\psi}_{t}$ is no longer a local symplectomorphism ; all other properties still hold.

Since by definition of $c$ the submanifolds $W_{t, k}$ are trivial over the considered balls, it follows from the implicit function theorem that $W_{t, k}$ can be parametrized around $x_{t}$ in the chosen coordinates as the set of points of the form $\tilde{\psi}_{t}\left(z, \tau_{t}(z)\right)$ for $z \in \mathbb{C}^{n-r}$, where $\tau_{t}: \mathbb{C}^{n-r} \rightarrow \mathbb{C}^{r}$ satisfies $\tau_{t}(0)=0$ and $\nabla \tau_{t}(0)=O\left(k^{-1 / 2}\right)$. The derivatives of $\tau_{t}$ can be easily computed, since it is characterized by the equation

$$
w_{t, k}\left(\tilde{\psi}_{t}\left(z, \tau_{t}(z)\right)\right)=0 .
$$

Notice that it follows from the transversality to 0 of $w_{t, k}$ that $\left.\mid \nabla w_{t, k} \circ d \tilde{\psi}_{t}(v)\right) \mid$ is larger than a constant times $k^{1 / 2}|v|$ for all $v \in 0 \times \mathbb{C}^{r}$. Combining this estimate with the bounds on the derivatives of $w_{t, k}$ given by asymptotic holomorphicity and the above bounds on those of $\tilde{\psi}_{t}$, one gets the following estimates for $\tau_{t}$ over the ball $\hat{B}$ :

$$
\left|\bar{\partial} \tau_{t}\right|=O\left(k^{-1 / 2}\right), \quad\left|\nabla \tau_{t}\right|=O(1), \quad\left|\nabla \bar{\partial} \tau_{t}\right|=O(1), \quad\left|\nabla \nabla \tau_{t}\right|=O\left(k^{1 / 2}\right) .
$$

It is then clear that $\theta_{t}(z)=\tilde{\psi}_{t}\left(z, \tau_{t}(z)\right)$ satisfies all the required properties.

Now that a local identification between $W_{t, k}$ and $\mathbb{C}^{n-r}$ is available, we define the restricted sections $\hat{s}_{t, k, x}(z)=s_{t, k, x}\left(\theta_{t}(z)\right)$ and $\hat{\sigma}_{t, k, j}(z)=\sigma_{t, k, j}\left(\theta_{t}(z)\right)$. Since $s_{t, k, x}$ and $\sigma_{t, k, j}$ are both asymptotically holomorphic, the estimates on $\theta_{t}$ imply that $\hat{s}_{t, k, x}$ and $\hat{\sigma}_{t, k, j}$, as sections of the pull-back of $L^{k}$ over the ball $\hat{B}$, are also asymptotically holomorphic. Furthermore, they clearly depend continuously on $t \in I$, and $\hat{s}_{t, k, x}$ remains larger than a fixed constant $c_{s}>0$ over $\hat{B}$. We can then define the complex-valued functions $f_{t, k, x}=\hat{\sigma}_{t, k, j} / \hat{s}_{t, k, x}$ over $\hat{B}$, which are clearly asymptotically holomorphic too. 
After dilation of $\hat{B}$ by a factor of $3 c . k^{1 / 2}$, all hypotheses of Proposition 3 are satisfied with $\delta$ as small as desired, provided that $k$ is large enough. Indeed, the asymptotic holomorphicity of $f_{t, k, x}$ implies that, for large $k$, the antiholomorphic part of the function over the dilated ball is smaller than $\sigma=\delta .\left(\log \delta^{-1}\right)^{-p}$. So the local result implies that there exist complex numbers $c_{t, k, x}$ of norm less than $\delta$ and depending continuously on $t \in I$, such that the functions $f_{t, k, x}-c_{t, k, x}$ are $\sigma$-transverse to 0 over the ball $\hat{B}^{-}$of radius $3 c . k^{-1 / 2}$ around 0 in $\mathbb{C}^{n-r}$. We now notice that the sections $\hat{g}_{t, k, x}=\hat{\sigma}_{t, k, j}-c_{t, k, x} \hat{s}_{t, k, x}$, which clearly depend continuously on $t$ and are asymptotically holomorphic, are $\sigma^{\prime}$-transverse to 0 over $\hat{B}^{-}$, for some $\sigma^{\prime}$ differing from $\sigma$ by at most a constant factor. Indeed,

$$
\nabla \hat{g}_{t, k, x}=\nabla\left(\hat{s}_{t, k, x}\left(f_{t, k, x}-c_{t, k, x}\right)\right)=\hat{s}_{t, k, x} \nabla f_{t, k, x}-\left(f_{t, k, x}-c_{t, k, x}\right) \nabla \hat{s}_{t, k, x} .
$$

Wherever $\hat{g}_{t, k, x}$ is very small, so is $f_{t, k, x}-c_{t, k, x}$, and $\nabla f_{t, k, x}$ is thus large. Since $\hat{s}_{t, k, x}$ remains larger than some $c_{s}>0$ and $\nabla \hat{s}_{t, k, x}$ is bounded by a constant times $k^{1 / 2}$, it follows that $\nabla \hat{g}_{t, k, x}$ is large wherever $\hat{g}_{t, k, x}$ is very small. Putting the right constants in the right places, one easily checks that $\hat{g}_{t, k, x}$ is $\sigma^{\prime}$-transverse to 0 with $\sigma / \sigma^{\prime}$ bounded by a fixed constant.

We now notice that the restrictions to $W_{t, k}$ of the sections $g_{t, k, x}=\sigma_{t, k, j}-$ $c_{t, k, x} s_{t, k, x}$ of $L^{k}$ over $U$, which clearly are asymptotically $J_{t}$-holomorphic and depend continuously and $t$, are also $\sigma^{\prime \prime}$-transverse to 0 over $\hat{B}_{t}$ for some $\sigma^{\prime \prime}$ differing from $\sigma^{\prime}$ by at most a constant factor. Indeed, $\hat{B}_{t}$ is contained in the set of all points of the form $\theta_{t}(z)$ for $z \in \hat{B}^{-}$, and

$$
g_{t, k, x}\left(\theta_{t}(z)\right)=\hat{\sigma}_{t, k, j}(z)-c_{t, k, x} \hat{s}_{t, k, x}(z)=\hat{g}_{t, k, x}(z),
$$

so wherever $g_{t, k, x}$ is smaller than $\sigma^{\prime}$, the derivative of $\hat{g}_{t, k, x}$ is larger than $\sigma^{\prime} . k^{1 / 2}$, and since $\nabla \theta_{t}$ is bounded by a fixed constant, $\nabla g_{t, k, x}$ is large too.

Next we extend the definition of $c_{t, k, x}$ to all $t \in T$, in the case of $T=[0,1]$, since we have defined it only over the components of $\Omega$. However when $t \notin \Omega$, $W_{t, k}$ does not meet the ball $B_{x}$, so that there is no transversality requirement. Thus the only constraints are that $c_{t, k, x}$ must depend continuously on $t$ and remain smaller than $\delta$ for all $t$. These conditions are easy to satisfy, so we have proved the following :

Lemma 5. For all large $k$ there exist complex numbers $c_{t, k, x}$ smaller than $\delta$ and depending continuously on $t \in T$ such that the restriction to $W_{t, k}$ of $\sigma_{t, k, j}-c_{t, k, x} s_{t, k, x}$ is $\sigma^{\prime \prime}$-transverse to 0 over $W_{t, k} \cap B_{x}$. Furthermore, for some constant $p^{\prime}$ depending only on the dimension, $\sigma^{\prime \prime}$ is at least $\delta .\left(\log \delta^{-1}\right)^{-p^{\prime}}$.

3.5. Constructing $\sigma_{t, k, j+1}$ from $\sigma_{t, k, j}$. We can now define the sections $\sigma_{t, k, j+1}$ of $L^{k}$ over $U$ by

$$
\sigma_{t, k, j+1}=\sigma_{t, k, j}-\sum_{x \in S_{j}} c_{t, k, x} s_{t, k, x}
$$

Clearly the sections $\sigma_{t, k, j+1}$ are asymptotically holomorphic and depend continuously on $t \in T$. Furthermore, any two points in $S_{j}$ are distant 
of at least $D \cdot k^{-1 / 2}$ with $D>0$, so the total size of the perturbation is bounded by a fixed multiple of $\delta$. So, choosing $\delta$ smaller than $\eta_{j}$ over a constant factor (recall that $\eta_{j}$ is the transversality estimate of the previous step of the iterative process), we can ensure that $\left|\sigma_{t, k, j+1}-\sigma_{t, k, j}\right|<\frac{\eta_{j}}{2}$ and $\left|\nabla \sigma_{t, k, j+1}-\nabla \sigma_{t, k, j}\right|<\frac{\eta_{j}}{2} k^{1 / 2}$. As a direct consequence, the restriction to $W_{t, k}$ of $\sigma_{t, k, j+1}$ is $\frac{\eta_{j}}{2}$-transverse to 0 wherever the restriction of $\sigma_{t, k, j}$ is $\eta_{j^{-}}$ transverse to 0 , including over $W_{t, k} \cap \mathcal{N}_{j}$ (recall that $\mathcal{N}_{j}=\bigcup_{i<j} \bigcup_{x \in S_{i}} B_{x}$ ).

Letting $\eta_{j+1}=\frac{1}{2} \sigma^{\prime \prime}$, it is known that for all $x \in S_{j}$ the restriction of $\sigma_{t, k, j}-c_{t, k, x} s_{t, k, x}$ to $W_{t, k}$ is $2 \eta_{j+1}$-transverse to 0 over $B_{x} \cap W_{t, k}$. So, in order to prove that the restriction to $W_{t, k}$ of $\sigma_{t, k, j+1}$ is $\eta_{j+1}$-transverse to 0 over $W_{t, k} \cap \mathcal{N}_{j+1}$, it is sufficient to check that given $x \in S_{j}$, over $B_{x}$, the sum of the perturbations corresponding to all points $y \in S_{j}$ distinct from $x$ is smaller than $\eta_{j+1}$, and the sum of their derivatives is smaller than $\eta_{j+1} k^{1 / 2}$. In other words, since several contributions were added at the same time (one at each point of $S_{j}$ ), we have to make sure that they cannot interfere.

This is where the parameter $D$ (minimum distance between two points in $S_{j}$ ) is important : indeed, over $B_{x}$, by Lemma 3 , each of the contributions of the other points in $S_{j}$ is at most of the order of $\delta \cdot \exp \left(-\lambda D^{2}\right)$, and the sum of these terms is $O\left(\eta_{j} \cdot \exp \left(-\lambda D^{2}\right)\right)$. Similarly, the derivative of that sum is $O\left(\eta_{j} \cdot \exp \left(-\lambda D^{2}\right) \cdot k^{1 / 2}\right)$. So the requirement that the sum of the contributions of all points of $S_{j}$ distinct from $x$ be smaller than $\eta_{j+1}$ corresponds to an inequality of the form $K_{0} \exp \left(-\lambda D^{2}\right)<\eta_{j+1} / \eta_{j}$, where $K_{0}$ is a fixed constant depending only on the geometry of $X$. Recalling that $\eta_{j+1}$ is no smaller than $\eta_{j} \cdot \log \left(\eta_{j}^{-1}\right)^{-P}$ for some fixed integer $P$, the required inequality is

$$
\exp \left(\lambda D^{2}\right)>K_{0} \cdot \log \left(\eta_{j}^{-1}\right)^{P}
$$

This inequality, which does not depend on $k$, must be satisfied by every $\eta_{j}$, for each of the $N$ steps of the process.

To check that the condition on $D$ can be enforced at all steps, we must recall that the number of steps in the process is $N=O\left(D^{2 n}\right)$, and study the sequence $\left(\eta_{j}\right)$ given by a fixed $\eta_{0}>0$ and the inductive definition described above. It can be shown (see Lemma 24 of [1]) that the sequence $\left(\eta_{j}\right)$ satisfies for all $j$ a bound of the type $\log \left(\eta_{j}^{-1}\right)=O(j \cdot \log (j))$. It follows that $\log \left(\eta_{N}^{-1}\right)^{P}=O\left(D^{2 n P} \cdot \log \left(D^{2 n}\right)^{P}\right)$, which is clearly subexponential : a choice of sufficiently large $D$ thus ensures that the required inequality holds at all steps. So the inductive process described above is valid, and leads to sections $\tilde{\sigma}_{t, k}=\sigma_{t, k, N}$ which are asymptotically $J_{t}$-holomorphic, depend continuously on $t$, and whose restrictions to $W_{t, k}$ are $\hat{\eta}$-transverse to 0 over $U_{k}^{-}$for $\hat{\eta}=\eta_{N}$. Furthermore, $\tilde{\sigma}_{t, k}$ is equal to $\sigma_{t, k}$ near the boundary of $U$ because we only added a linear combination of sections $s_{t, k, x}$ for $x \in U_{k}^{-}$, and $s_{t, k, x}$ vanishes by construction outside of the ball of radius $k^{-1 / 3}$ around $x$. Moreover, $\tilde{\sigma}_{t, k}$ differs from $\sigma_{t, k}$ by at most $\sum_{j} \eta_{j}$, which is less than $2 \eta_{0}=\epsilon$. So to complete 
the proof of Theorem 3 we only have to show that the transversality result on $\tilde{\sigma}_{t, k \mid W_{t, k}}$ implies the transversality to 0 of $\left(w_{t, k}+\tilde{\sigma}_{t, k}\right)$ over $U_{k}^{=}$.

3.6. Transversality to 0 over $U_{k}^{=}$. At a point $x \in W_{t, k} \cap U_{k}^{-}$where $\left|\tilde{\sigma}_{t, k}\right|<$ $\hat{\eta}$, we know that $\nabla w_{t, k}$ is surjective and vanishes in all directions tangential to $W_{t, k}$, while $\nabla \tilde{\sigma}_{t, k}$ has a tangential component larger than $\hat{\eta} \cdot k^{1 / 2}$. It follows that $\nabla\left(w_{t, k}+\tilde{\sigma}_{t, k}\right)$ is surjective. We now construct a right inverse $R:\left(E_{x} \oplus \mathbb{C}\right) \otimes L_{x}^{k} \rightarrow T_{x} X$ whose norm is $O\left(k^{-1 / 2}\right)$.

Considering a unit length element $u$ of $L_{x}^{k}$, there exists a vector $\hat{u} \in T_{x} W_{t, k}$ of norm at most $\left(\hat{\eta} \cdot k^{1 / 2}\right)^{-1}$ such that $\nabla \tilde{\sigma}_{t, k}(\hat{u})=u$. Clearly $\nabla w_{t, k}(\hat{u})=0$ because $\hat{u} \in T_{x} W_{t, k}$, so we define $R(u)=\hat{u}$. Now consider an orthonormal frame $\left(v_{i}\right)$ in $E_{x} \otimes L_{x}^{k}$. It follows from the $\eta$-transversality to 0 of $w_{t, k}$ that $\nabla_{x} w_{t, k}$ has a right inverse of norm smaller than $\left(\eta \cdot k^{1 / 2}\right)^{-1}$, so we obtain vectors $\hat{v}_{i}$ in $T_{x} X$ such that $\nabla w_{t, k}\left(\hat{v}_{i}\right)=v_{i}$ and $\left|\hat{v}_{i}\right|<\left(\eta \cdot k^{1 / 2}\right)^{-1}$. There exist coefficients $\lambda_{i}$ such that $\nabla \tilde{\sigma}_{t, k}\left(\hat{v}_{i}\right)=\lambda_{i}$. u, with $\left|\lambda_{i}\right|<C . k^{1 / 2} .\left|\hat{v}_{i}\right|<C . \eta^{-1}$, for some constant $C$ such that $\left|\nabla \tilde{\sigma}_{t, k}\right|<C . k^{1 / 2}$ everywhere. So we define $R\left(v_{i}\right)=\hat{v}_{i}-\lambda_{i} \hat{u}$, which completes the determination of $R$.

The norm of $R$ is, by construction, smaller than $K \cdot k^{-1 / 2}$ for some $K$ depending only on the constants above $(C, \eta$ and $\hat{\eta})$. We thus know that $\nabla\left(w_{t, k}+\tilde{\sigma}_{t, k}\right)$ has a right inverse smaller than $K . k^{-1 / 2}$ at any point of $W_{t, k} \cap U_{k}^{-}$where $\left|\tilde{\sigma}_{t, k}\right|<\hat{\eta}$. Furthermore we know, from the definition of asymptotic holomorphicity, that $\left|\nabla \nabla\left(w_{t, k}+\tilde{\sigma}_{t, k}\right)\right|<K^{\prime} . k$ for some constant $K^{\prime}$.

Consider a point $x$ of $U_{k}^{=}$where $\left|w_{t, k}\right|$ and $\left|\tilde{\sigma}_{t, k}\right|$ are both smaller than some $\alpha$ which is simultaneously smaller than $\frac{\hat{\eta}}{2}, \frac{\eta \hat{\eta}}{2 C}$ and $\frac{\eta}{2 K K^{\prime}}$. From the $\eta$-transversality to 0 of $w_{t, k}$, we know that $\nabla w_{t, k}$ is surjective at $x$ and has a right inverse smaller than $\left(\eta \cdot k^{1 / 2}\right)^{-1}$. Since the connection $\nabla$ is unitary, applying the right inverse to $w_{t, k}$ itself, we can follow the downward gradient flow of $\left|w_{t, k}\right|$, and we are certain to reach a point $y$ of $W_{t, k}$ at a distance $d$ from the starting point $x$ no larger than $\alpha .\left(\eta \cdot k^{1 / 2}\right)^{-1}$, which is simultaneously smaller than $\frac{1}{2 K K^{\prime}} \cdot k^{-1 / 2}$ and $\frac{\hat{\eta}}{2 C} \cdot k^{-1 / 2}$. Furthermore if $k$ is large enough, $d<2 k^{-1 / 3}$ so that $y \in U_{k}^{-}$.

Since $\left|\nabla \tilde{\sigma}_{t, k}\right|<C . k^{1 / 2}$ everywhere, $\left|\tilde{\sigma}_{t, k}(y)\right|-\left|\tilde{\sigma}_{t, k}(x)\right|<C . k^{1 / 2} . d<\frac{\hat{\eta}}{2}$, so that $\left|\tilde{\sigma}_{t, k}(y)\right|<\hat{\eta}$, and the previous results apply at $y$. Also, since the second derivatives are bounded by $K^{\prime} . k$ everywhere, $\nabla_{x}\left(w_{t, k}+\tilde{\sigma}_{t, k}\right)$ differs from $\nabla_{y}\left(w_{t, k}+\tilde{\sigma}_{t, k}\right)$ by at most $K^{\prime} k . d$, which is smaller than $\frac{1}{2 K} \cdot k^{1 / 2}$, so that it is still surjective and admits a right inverse of norm $O\left(k^{-1 / 2}\right)$. From this we infer immediately that $\left(w_{t, k}+\tilde{\sigma}_{t, k}\right)$ is transverse to 0 over all of $U_{k}^{=}$, and the proof of Theorem 3 is complete.

\section{The MAIn Result}

4.1. Proof of Theorem 2. Theorem 2 follows from Theorem 3 by a simple induction argument. Indeed, to obtain asymptotically holomorphic sections 
of $E \otimes L^{k}$ which are transverse to 0 over $X$ for any vector bundle $E$, we start from the fact that $E$ is locally trivial, so that there exists a finite covering of $X$ by $N$ open subsets $U_{j}$ such that $E$ is a trivial bundle on a small neighborhood of each $U_{j}$. We start initially from the sections $s_{t, k, 0}=s_{t, k}$ of $E \otimes L^{k}$, and proceed iteratively, assuming at the beginning of the $j$-th step that we have constructed, for all large $k$, asymptotically holomorphic sections $s_{t, k, j}$ of $E \otimes L^{k}$ which are $\eta_{j}$-transverse to 0 on $\bigcup_{i<j} U_{i}$ for some $\eta_{j}>0$ and differ from $s_{t, k}$ by at most $j \epsilon / N$.

Over a small neighborhood of $U_{j}$, we trivialize $E \simeq \mathbb{\mathbb { C }}^{r}$ and decompose the sections $s_{t, k, j}$ into their $r$ components for this trivialization. Recall that, in order to define the connections on $E \otimes L^{k}$ for which asymptotic holomorphicity and transversality to 0 are expected, we have used a hermitian connection $\nabla^{E}$ on $E$. Because $X$ is compact the connection 1-form of $\nabla^{E}$ in the chosen trivializations can be safely assumed to be bounded by a fixed constant. It follows that, up to a change in the constants, asymptotic holomorphicity and transversality to 0 over $U_{j}$ with respect to the connections on $E \otimes L^{k}$ induced by $\nabla^{E}$ and $\nabla^{L}$ are equivalent to asymptotic holomorphicity and transversality to 0 with respect to the connections induced by $\nabla^{L}$ and the trivial connection on $E$ in the chosen trivialization. So we actually do not have to worry about $\nabla^{E}$.

Now let $\alpha$ be a constant smaller than both $\epsilon / r N$ and $\eta_{j} / 2 r$. First, using Theorem 3, we perturb the first component of $s_{t, k, j}$ over a neighborhood of $U_{j}$ by at most $\alpha$ to make it transverse to 0 over a slightly smaller neighborhood. Next, using again Theorem 3, we perturb the second component by at most $\alpha$ so that the sum of the two first components is transverse to 0 , and so on, perturbing the $i$-th component by at most $\alpha$ to make the sum of the $i$ first components transverse to 0 . The result of this process is a family of asymptotically $J_{t}$-holomorphic sections $s_{t, k, j+1}$ of $E \otimes L^{k}$ which are transverse to 0 over $U_{j}$. Furthermore, since the total perturbation is smaller than $r \alpha \leq \eta_{j} / 2$, transversality to 0 still holds over $U_{i}$ for $i<j$, so that the hypotheses of the next step are satisfied. The construction thus leads to sections $\sigma_{t, k}=s_{t, k, N}$ which are transverse to 0 over all of $X$. Since at each of the $N$ steps the total perturbation is less than $\epsilon / N$, the sections $\sigma_{t, k}$ differ from $s_{t, k}$ by less than $\epsilon$, and Theorem 2 is proved.

4.2. Symplectic isotopies. We now give the remaining part of the proof of Corollary 2, namely the following statement :

Proposition 4. let $\left(W_{t}\right)_{t \in[0,1]}$ be a family of symplectic submanifolds in $X$. Then there exist symplectomorphisms $\Phi_{t}: X \rightarrow X$ depending continuously on $t$, such that $\Phi_{0}=\operatorname{Id}$ and $\Phi_{t}\left(W_{0}\right)=W_{t}$.

The following strategy of proof, based on Moser's ideas, was suggested to me by M. Gromov. The reader unfamiliar with these techniques may use [4] (pp. 91-101) as a reference.

It follows immediately from Moser's stability theorem that there exists a continuous family of symplectomorphisms $\phi_{t}:\left(W_{0}, \omega_{\mid W_{0}}\right) \rightarrow\left(W_{t}, \omega_{\mid W_{t}}\right)$. 
Since the symplectic normal bundles to $W_{t}$ are all isomorphic, Weinstein's symplectic neighborhood theorem allows one to extend these maps to symplectomorphisms $\psi_{t}: U_{0} \rightarrow U_{t}$ such that $\psi_{t}\left(W_{0}\right)=W_{t}$, where $U_{t}$ is a small tubular neighborhood of $W_{t}$ for all $t$.

Let $\rho_{t}$ be any family of diffeomorphisms of $X$ extending $\psi_{t}$. Let $\omega_{t}=\rho_{t}^{*} \omega$ and $\Omega_{t}=-d \omega_{t} / d t$. We want to find vector fields $\xi_{t}$ on $X$ such that the 1-forms $\alpha_{t}=\iota_{\xi_{t}} \omega_{t}$ satisfy $d \alpha_{t}=\Omega_{t}$ and such that $\xi_{t}$ is tangent to $W_{0}$ at any point of $W_{0}$. If this is possible, then define diffeomorphisms $\Psi_{t}$ as the flow of the vector fields $\xi_{t}$, and notice that

$$
\frac{d}{d t}\left(\Psi_{t}^{*} \rho_{t}^{*} \omega\right)=\Psi_{t}^{*}\left(\frac{d}{d t}\left(\rho_{t}^{*} \omega\right)+L_{\xi_{t}}\left(\rho_{t}^{*} \omega\right)\right)=\Psi_{t}^{*}\left(-\Omega_{t}+d \iota_{\xi_{t}} \omega_{t}\right)=0 .
$$

So the diffeomorphisms $\rho_{t} \circ \Psi_{t}$ are actually symplectomorphisms of $X$. Furthermore $\Psi_{t}$ preserves $W_{0}$ by construction, so $\rho_{t} \circ \Psi_{t}$ maps $W_{0}$ to $W_{t}$, thus giving the desired result.

So we are left with the problem of finding $\xi_{t}$, or equivalently $\alpha_{t}$, such that $d \alpha_{t}=\Omega_{t}$ and $\xi_{t \mid W_{0}}$ is tangent to $W_{0}$. Note that, since $\rho_{t}$ extends the symplectomorphisms $\psi_{t}$, one has $\omega_{t}=\omega$ and $\Omega_{t}=0$ over $U_{0}$. It follows that the condition on $\xi_{t \mid W_{0}}$ is equivalent to the requirement that at any point $x \in W_{0}$, the $\omega$-symplectic orthogonal $N_{x} W_{0}$ to $T_{x} W_{0}$ lies in the kernel of the 1-form $\alpha_{t}$.

Since the closed 2-forms $\omega_{t}$ are all cohomologous, one has $\left[\Omega_{t}\right]=0$ in $H^{2}(X, \mathbb{R})$, so there exist 1 -forms $\beta_{t}$ on $X$ such that $d \beta_{t}=\Omega_{t}$. Remark that, although $\Omega_{t}=0$ over $U_{0}$, one cannot ensure that $\beta_{t \mid U_{0}}=0$ unless the class $\left[\Omega_{t}\right]$ also vanishes in the relative cohomology group $H^{2}\left(X, U_{0} ; \mathbb{R}\right)$. So we need to work a little more to find the proper 1-forms $\alpha_{t}$.

Over $U_{0}$ one has $d \beta_{t}=\Omega_{t}=0$, so $\beta_{t}$ defines a class in $H^{1}\left(U_{0}, \mathbb{R}\right)$. By further restriction, the forms $\beta_{t \mid W_{0}}$ are also closed 1-forms on $W_{0}$. Let $\pi$ be a projection map $U_{0} \rightarrow W_{0}$ such that at any point $x \in W_{0}$ the tangent space to $\pi^{-1}(x)$ is the symplectic normal space $N_{x} W_{0}$, and let $\gamma_{t}=\pi^{*}\left(\beta_{t \mid W_{0}}\right)$. First we notice that, by construction, the 1 -form $\gamma_{t}$ is closed over $U_{0}$, and at any point $x \in W_{0}$ the space $N_{x} W_{0}$ lies in the kernel of $\gamma_{t}$. Furthermore the composition of $\pi^{*}$ and the restriction map induces the identity map over $H^{1}\left(U_{0}, \mathbb{R}\right)$, so $\left[\gamma_{t}\right]=\left[\beta_{t \mid U_{0}}\right]$ in $H^{1}\left(U_{0}, \mathbb{R}\right)$. Therefore there exist functions $f_{t}$ over $U_{0}$ such that $\gamma_{t}=\beta_{t}+d f_{t}$ at any point of $U_{0}$.

Let $g_{t}$ be any smooth functions over $X$ extending $f_{t}$, and let $\alpha_{t}=\beta_{t}+d g_{t}$. The 1 -forms $\alpha_{t}$ satisfy $d \alpha_{t}=d \beta_{t}=\Omega_{t}$, and since $\alpha_{t \mid U_{0}}=\gamma_{t}$ the space $N_{x} W_{0}$ also lies in the kernel of $\alpha_{t}$ at any $x \in W_{0}$. So Proposition 4 is proved.

\section{Properties of the COnstructed submanifolds}

5.1. Proof of Proposition 2. This proof is based on that of a similar result obtained by Donaldson [1] for the submanifolds obtained from Theorem 1 $(r=1)$. The result comes from a Morse theory argument, as described in [1]. Indeed, consider the real valued function $f=\log |s|^{2}$ over $X-W$ (where $\left.W=s^{-1}(0)\right)$. We only have to show that, if $k$ is large enough, all its critical 
points are of index at least $n-r+1$. For this purpose, let $x$ be a critical point of $f$, and let us compute the derivative $\bar{\partial} \partial f$ at $x$.

First we notice that $x$ is also a critical point of $|s|^{2}$, so that $s$ itself is not in the image of $\nabla_{x} s$. Recalling that $s$ is $\eta$-transverse to 0 for some $\eta>0$, it follows that $\nabla_{x} s$ is not surjective and thus $|s(x)| \geq \eta$.

Recalling that the scalar product is linear in the first variable and antilinear in the second variable, we compute the derivative

$$
\partial \log |s|^{2}=\frac{1}{|s|^{2}}(\langle\partial s, s\rangle+\langle s, \bar{\partial} s\rangle)
$$

which equals zero at $x$. A first consequence is that, at $x,|\langle\partial s, s\rangle|=$ $|\langle\bar{\partial} s, s\rangle|<C|s|$, where $C$ is a constant bounding $\bar{\partial} s$ independently of $k$.

A second derivation, omitting the quantities that vanish at a critical point, yields that, at $x$,

$$
\bar{\partial} \partial \log |s|^{2}=\frac{1}{|s|^{2}}(\langle\bar{\partial} \partial s, s\rangle-\langle\partial s, \partial s\rangle+\langle\bar{\partial} s, \bar{\partial} s\rangle+\langle s, \partial \bar{\partial} s\rangle) .
$$

Recall that $\bar{\partial} \partial+\partial \bar{\partial}$ is equal to the part of type $(1,1)$ of the curvature of the bundle $E \otimes L^{k}$. This is equal to $-i k \omega \otimes \mathrm{Id}+R$, where $R$ is the part of type $(1,1)$ of the curvature of $E$, so that at $x$,

$\bar{\partial} \partial \log |s|^{2}=-i k \omega+\frac{1}{|s|^{2}}(\langle R . s, s\rangle-\langle\partial \bar{\partial} s, s\rangle+\langle s, \partial \bar{\partial} s\rangle-\langle\partial s, \partial s\rangle+\langle\bar{\partial} s, \bar{\partial} s\rangle)$.

To go further, we have to restrict our choice of vectors to a subspace of the tangent space $T_{x} X$ at $x$. Call $\Theta$ the space of all vectors $v$ in $T_{x} X$ such that $\partial s(v)$ belongs to the complex line generated by $s$ in $\left(E \otimes L^{k}\right)_{x}$. The subspace $\Theta$ of $T_{x} X$ is clearly stable by the almost-complex structure, and its complex dimension is at least $n-r+1$. For any vector $v \in \Theta$, $|\langle\partial s(v), s\rangle|=|\partial s(v)| .|s|$ is smaller than $|v| \cdot|\langle\partial s, s\rangle|<C|v| \cdot|s|$ where $C$ is the same constant as above, so that $\partial s$ is $O(1)$ over $\Theta$.

Since $\bar{\partial} s=O(1)$ and $\partial \bar{\partial} s=O\left(k^{1 / 2}\right)$ because of asymptotic holomorphicity, it is now known that the restriction to $\Theta$ of $\bar{\partial} \partial \log |s|^{2}$ is equal to $-i k \omega+O\left(k^{1 / 2}\right)$. It follows that, for all large $k$, given any unit length vector $u \in \Theta$, the quantity $-2 i \bar{\partial} \partial f(u, J u)$, which equals $H_{f}(u)+H_{f}(J u)$ where $H_{f}$ is the Hessian of $f$ at $x$, is negative. If the index of the critical point at $x$ were less than $n-r+1$, there would exist a subspace $P \subset T_{x} X$ of real dimension at least $n+r$ over which $H_{f}$ is non-negative, and the subspace $P \cap J P$ of real dimension at least $2 r$ would necessarily intersect non-trivially with $\Theta$ whose real dimension is at least $2 n-2 r+2$, contradicting the previous remark. The index of the critical point $x$ of $f$ is thus at least $n-r+1$.

A standard Morse theory argument then implies that the inclusion $W \rightarrow$ $X$ induces an isomorphism on all homotopy (and homology) groups up to $\pi_{n-r-1}$ (resp. $H_{n-r-1}$ ), and a surjection on $\pi_{n-r}$ (resp. $H_{n-r}$ ), which completes the proof of Proposition 2. 
5.2. Homology and Chern numbers of the submanifolds. Proposition 2 allows one to compute the middle-dimensional Betti number $b_{n-r}=$ $\operatorname{dim} H_{n-r}\left(W_{k}, \mathbb{R}\right)$ of the constructed submanifolds. Indeed the tangent bundle $T W_{k}$ and the normal bundle $N W_{k}$ (isomorphic to the restriction to $W_{k}$ of $E \otimes L^{k}$ ) are both symplectic vector bundles over $W_{k}$. So it is well-known (see e.g. [4], p. 67) that they admit underlying structures of complex vector bundles, uniquely determined up to homotopy (in our case there exist $J$-stable subspaces in $T X$ very close to $T W_{k}$ and $N W_{k}$, so after a small deformation one can think of these complex structures as induced by $J$ ). Furthermore one has $T W_{k} \oplus N W_{k} \simeq T X_{\mid W_{k}}$. It follows that, calling $i$ the inclusion map $W_{k} \rightarrow X$, the Chern classes of the bundle $T W_{k}$ can be computed from the relation

$$
i^{*} c(T X)=i^{*} c\left(E \otimes L^{k}\right) . c\left(T W_{k}\right) .
$$

Since $c_{n-r}\left(T W_{k}\right) \cdot\left[W_{k}\right]$ is equal to the Euler-Poincaré characteristic of $W_{k}$, and since the spaces $H_{i}\left(W_{k}, \mathbb{R}\right)$ have the same dimension as $H_{i}(X, \mathbb{R})$ for $i<n-r$, the dimension of $H_{n-r}\left(W_{k}, \mathbb{R}\right)$ follows immediately.

For further computations, we need an estimate on this dimension :

Proposition 5. For any sequence of symplectic submanifolds $W_{k} \subset X$ of real codimension $2 r$ obtained as the zero sets of asymptotically holomorphic sections of $E \otimes L^{k}$ which are transverse to 0 , the Chern classes of $W_{k}$ are given by

$$
c_{l}\left(T W_{k}\right)=(-1)^{l}\left(\begin{array}{c}
r+l-1 \\
l
\end{array}\right)(k \hat{\omega})^{l}+O\left(k^{l-1}\right),
$$

where $\hat{\omega}$ denotes the class of $\frac{\omega}{2 \pi}$ in the cohomology of $W_{k}$.

This can be proved by induction on $l$, starting from $c_{0}\left(T W_{k}\right)=1$, since the above equality implies that

$$
c_{l}\left(T W_{k}\right)=i^{*} c_{l}(T X)-\sum_{j=0}^{l-1} i^{*} c_{l-j}\left(E \otimes L^{k}\right) . c_{j}\left(T W_{k}\right) .
$$

It can be checked that $i^{*} c_{l-j}\left(E \otimes L^{k}\right)=\left(\begin{array}{c}r \\ l-j\end{array}\right)(k \hat{\omega})^{l-j}+O\left(k^{l-j-1}\right)$, so that the result follows from a combinatorial calculation showing that the quantity $\sum_{j=0}^{l}(-1)^{j}\left(\begin{array}{c}r \\ l-j\end{array}\right)\left(\begin{array}{c}r+j-1 \\ j\end{array}\right)$ is equal to 0.

Since $\left[W_{k}\right]$ is Poincaré dual in $X$ to $c_{r}\left(E \otimes L^{k}\right)$, Proposition 5 yields that $\chi\left(W_{k}\right)=c_{n-r}\left(T W_{k}\right) \cdot\left[W_{k}\right]=(-1)^{n-r}\left(\begin{array}{l}n-1 \\ n-r\end{array}\right)(k \hat{\omega})^{n-r} \cdot(k \hat{\omega})^{r}+O\left(k^{n-1}\right)$. Finally, Proposition 2 implies that $\chi\left(W_{k}\right)=(-1)^{n-r} \operatorname{dim} H_{n-r}\left(W_{k}, \mathbb{R}\right)+O(1)$, so that

$$
\operatorname{dim} H_{n-r}\left(W_{k}, \mathbb{R}\right)=\left(\begin{array}{l}
n-1 \\
n-r
\end{array}\right)\left[\frac{\omega}{2 \pi}\right]^{n} \cdot k^{n}+O\left(k^{n-1}\right) .
$$

5.3. Geometry of the submanifolds. Aside from the above topological information on the submanifolds, one can also try to characterize the geometry of $W_{k}$ inside $X$. We prove the following result, expressing the fact that the middle-dimensional homology of $W_{k}$ has many generators that are very "localized" around any given point of $X$ : 
Proposition 6. There exists a constant $C>0$ depending only on the geometry of the manifold $X$ with the following property : let $B$ be any ball of small enough radius $\rho>0$ in $X$. For any sequence of symplectic submanifolds $W_{k} \subset X$ of real codimension $2 r$ obtained as the zero sets of asymptotically holomorphic sections of $E \otimes L^{k}$ which are transverse to 0 , let $N_{k}(B)$ be the number of independent generators of $H_{n-r}\left(W_{k}, \mathbb{R}\right)$ which can be realized by cycles that are entirely included in $W_{k} \cap B$. Then, if $k$ is large enough, one has

$$
N_{k}(B)>C \cdot \rho^{2 n} \cdot \operatorname{dim} H_{n-r}\left(W_{k}, \mathbb{R}\right) .
$$

As a consequence, we can state that when $k$ becomes large the submanifolds $W_{k}$ tend to "fill out" all of $X$, since they must intersect non-trivially with any given ball.

The proof of Proposition 6 relies on the study of what happens when we perform a symplectic blow-up on the manifold $X$ inside the ball $B$. Recall that the blown-up manifold $\tilde{X}$ is endowed with a symplectic form $\tilde{\omega}$ which is equal to $\omega$ outside of $B$, and can be described inside $B$ using the following model on $\mathbb{C}^{n}$ around 0 : define on $\mathbb{C}^{n} \times\left(\mathbb{C}^{n}-\{0\}\right)$ the 2 -form

$$
\phi=i \partial \bar{\partial}\left(p_{1}^{*} \beta \cdot p_{2}^{*} \log \|\cdot\|^{2}\right),
$$

where $p_{1}$ is the projection map to $\mathbb{C}^{n}, \beta$ is a cut-off function around the blow-up point, and $p_{2}$ is the projection on the factor $\mathbb{C}^{n}-\{0\}$. The 2 -form $\phi$ projects to $\mathbb{C}^{n} \times \mathbb{C P}^{n-1}$, and after restriction to the graph of the blown-up manifold (i.e. the set of all $(x, y)$ such that $x$ belongs to the complex line in $\mathbb{C}^{n}$ defined by $y$ ) one obtains a closed 2-form whose restriction to the exceptional divisor is positive. Calling $\theta$ the 2 -form on $\tilde{X}$ supported in $B$ defined by this procedure, it can be checked that, if $\epsilon>0$ is small enough and $\pi$ is the projection map $\tilde{X} \rightarrow X$, the 2 -form $\tilde{\omega}=\pi^{*} \omega+\epsilon \theta$ is symplectic on $\tilde{X}$.

If we call $e \in H^{2}(\tilde{X}, \mathbb{Z})$ the Poincaré dual of the exceptional divisor, since its normal bundle is the inverse of the standard bundle over $\mathbb{C P}^{n-1}$, we have $(-e)^{n-1} \cdot e=1$, so that $e^{n}=(-1)^{n-1}$. Furthermore, the cohomology class of $\tilde{\omega}$ is given by $\left[\frac{\tilde{\omega}}{2 \pi}\right]=\pi^{*}\left[\frac{\omega}{2 \pi}\right]-\epsilon . e$. Now we consider the sections $s_{k}$ of $E \otimes L^{k}$ over $X$ which define $W_{k}$, and assuming $\epsilon^{-1}$ to be an integer we write $k=K+\tilde{k}$ with $0 \leq \tilde{k}<\epsilon^{-1}$ and $\epsilon K \in \mathbb{N}$. Notice that $\tilde{\omega}=\pi^{*} \omega$ outside $B$ and that we can safely choose a metric on $\tilde{X}$ with the same property. Considering that the line bundle $\tilde{L}^{K}$ on $\tilde{X}$ whose first Chern class is $K\left[\frac{\tilde{\omega}}{2 \pi}\right]$ is isomorphic to $\pi^{*} L^{K}$ over $\tilde{X}-B$, the sections $\pi^{*} s_{k}$ of $\pi^{*}\left(E \otimes L^{k}\right)=\pi^{*}\left(E \otimes L^{\tilde{k}}\right) \otimes \pi^{*} L^{K}$ obtained by pull-back of $s_{k}$ satisfy all desired conditions outside $B$, namely asymptotic holomorphicity and transversality to 0 . If we multiply $\pi^{*} s_{k}$ by a cut-off function equal to 1 over $\tilde{X}-B$ and vanishing over the support of $\theta$, we now obtain asymptotically holomorphic sections of $\pi^{*}\left(E \otimes L^{\tilde{k}}\right) \otimes \tilde{L}^{K}$ over $\tilde{X}$ which are transverse to 0 over $\tilde{X}-B$. So, if $K$ is large enough, we can use the construction described in Theorems 2 and 3 to perturb these sections over $B$ only to make them transverse to 0 over all of $\tilde{X}$. Since there 
are only finitely many values of $\tilde{k}$, the bounds on $K$ required for each $\tilde{k}$ translate as a single bound on $k$. Considering the zero sets of the resulting sections, we thus obtain symplectic submanifolds $\tilde{W}_{k} \subset \tilde{X}$ to which we can again apply Propositions 2 and 5 . The interesting remark is that, using the above estimate for $\operatorname{dim} H_{n-r}\left(\tilde{W}_{k}, \mathbb{R}\right)$, since $\left[\frac{\tilde{\omega}}{2 \pi}\right]^{n}=\left[\frac{\omega}{2 \pi}\right]^{n}-\epsilon^{n}$ (symplectic blowups decrease the symplectic volume), we get for all large $k$

$$
\operatorname{dim} H_{n-r}\left(\tilde{W}_{k}, \mathbb{R}\right)=\operatorname{dim} H_{n-r}\left(W_{k}, \mathbb{R}\right)-\epsilon^{n}\left(\begin{array}{c}
n-1 \\
n-r
\end{array}\right) k^{n}+O\left(k^{n-1}\right) .
$$

This means that we have decreased the dimension of $H_{n-r}\left(W_{k}, \mathbb{R}\right)$ by changing the picture only inside the ball $B$. To continue we need an estimate on the dependence of $\epsilon$ on the radius $\rho$ of the ball. The main constraint on $\epsilon$ is that $\epsilon \theta$ should be much smaller than $\pi^{*} \omega$ so that the perturbation does not affect the positivity of $\pi^{*} \omega$. The norm of $\theta$ is directly related to that of the second derivative $\partial \bar{\partial} \beta$ of the cut-off function $\beta$. Since the only constraint on $\beta$ is that it should be 0 outside $B$ and 1 near the blow-up point, an appropriate choice of $\beta$ leads to a bound of the type $|\partial \bar{\partial} \beta|=O\left(\rho^{-2}\right)$. It follows that $\epsilon$ can be chosen equal at least to a constant times $\rho^{2}$. So we obtain that, for a suitable value of $C$ and for all large enough $k$,

$$
\operatorname{dim} H_{n-r}\left(\tilde{W}_{k}, \mathbb{R}\right)<\left(1-2 C \rho^{2 n}\right) \operatorname{dim} H_{n-r}\left(W_{k}, \mathbb{R}\right) .
$$

Proposition 6 now follows immediately from the following general lemma by decomposing $W_{k}$ into $\left(W_{k}-B\right) \cup\left(W_{k} \cap B\right)$ and perturbing slightly $\rho$ if necessary so that the boundary of $B$ is transverse to $W_{k}$ :

Lemma 6. Let $W$ be a 2d-dimensional compact manifold which decomposes into two pieces $W=A \cup B$ glued along their common boundary $S$, which is a smooth codimension 1 submanifold in $W$. Assume that there exists a manifold $\tilde{W}$ which is identical to $W$ outside of $B$, and such that $\operatorname{dim} H_{d}(\tilde{W}, \mathbb{R}) \leq \operatorname{dim} H_{d}(W, \mathbb{R})-N$. Then there exists a $\frac{N}{2}$-dimensional subspace in $H_{d}(W, \mathbb{R})$ consisting of classes which can be represented by cycles contained in $B$.

To prove this lemma, let $H=H_{d}(W, \mathbb{R})$ and consider its subspaces $F$ consisting of all classes which can be represented by a cycle contained in $A$ and $G$ consisting of all classes representable in $B$. We have to show that $\operatorname{dim} G \geq \frac{N}{2}$. Let $G^{\perp}$ be the subspace of $H$ orthogonal to $G$ with respect to the intersection pairing, namely the set of classes which intersect trivially with all classes in $G$. We claim that $G^{\perp} \subset F+G$.

Indeed, let $\alpha$ be a cycle realizing a class in $G^{\perp}$. Subdividing $\alpha$ along its intersection with the common boundary $S$ of $A$ and $B$, we have $\alpha=$ $\alpha_{1}+\alpha_{2}$ where $\alpha_{1}$ and $\alpha_{2}$ are chains respectively in $A$ and $B$, such that $\partial \alpha_{1}=-\partial \alpha_{2}=\beta$ is a $(d-1)$-cycle contained in $S$. However $\beta$ intersects trivially with any $d$-cycle in $S$ since $\alpha$ intersects trivially with all cycles that have a representative in $B$. So the homology class represented by $\beta$ in $H_{d-1}(S, \mathbb{R})$ is trivial, and we have $\beta=\partial \gamma$ for some $d$-chain $\gamma$ in $S$. Writing $\alpha=\left(\alpha_{1}-\gamma\right)+\left(\alpha_{2}+\gamma\right)$ and shifting slightly the two copies of $\gamma$ on either side of $S$, we get that $[\alpha] \in F+G$. 
It follows that, if $F_{G}$ is a supplementary of $F \cap G$ in $F, \operatorname{dim} F_{G}+$ $\operatorname{dim} G=\operatorname{dim}(F+G)$ is larger than $\operatorname{dim} G^{\perp} \geq \operatorname{dim} H-\operatorname{dim} G$, so that $\operatorname{dim} G \geq \frac{1}{2}\left(\operatorname{dim} H-\operatorname{dim} F_{G}\right)$. Thus it only remains to show that $\operatorname{dim} F_{G} \leq$ $\operatorname{dim} H_{d}(\tilde{W}, \mathbb{R})$ to complete the proof of the lemma. To do this, we remark that the morphism $h: H_{d}(W ; \mathbb{R}) \rightarrow H_{d}(W, B ; \mathbb{R})$ in the relative homology sequence is injective on $F_{G}$, since its kernel is precisely $G$. However, if we define $\tilde{F}$ and $\tilde{G}$ inside $H_{d}(\tilde{W}, \mathbb{R})$ similarly to $F$ and $G$, the subspace $\tilde{F}_{\tilde{G}}$ similarly injects into $H_{d}(\tilde{W}, \tilde{B} ; \mathbb{R})$. Furthermore, the images of the two injections are both equal to the image of the morphism $H_{d}(A ; \mathbb{R}) \rightarrow H_{d}(A, S ; \mathbb{R})$ under the identification $H_{d}(\tilde{W}, \tilde{B} ; \mathbb{R}) \simeq H_{d}(A, S ; \mathbb{R}) \simeq H_{d}(W, B ; \mathbb{R})$, so that $\operatorname{dim} H_{d}(\tilde{W}, \mathbb{R}) \geq \operatorname{dim} \tilde{F}_{\tilde{G}}=\operatorname{dim} F_{G}$ and the proof is complete.

\section{Conclusion}

This paper has extended the field of applicability of the construction outlined by Donaldson [1] to more general vector bundles. It is in fact probable that similar methods can be used in other situations involving sequences of vector bundles whose curvatures become very positive.

The statement that, in spite of the high flexibility of the construction, the submanifolds obtained as zero sets of asymptotically holomorphic sections of $E \otimes L^{k}$ which are transverse to 0 are all isotopic for a given large enough $k$, has important consequences. Indeed, as suggested by Donaldson, it may allow the definition of relatively easily computable invariants of higher-dimensional symplectic manifolds from the topology of their submanifolds, for example from the Seiberg-Witten invariants of 4-dimensional submanifolds [5][6]. Furthermore, it facilitates the characterization of the topology of the constructed submanifolds in many cases, thus leading the way to many possibly new examples of symplectic manifolds.

\section{REFERENCES}

[1] S.K. Donaldson, Symplectic Submanifolds and Almost-complex Geometry, J. Diff. Geom. 44 (1996), 666-705.

[2] R.E. Gompf, A New Construction of Symplectic Manifolds, Ann. of Math. 142 (1995), 527-595.

[3] M. Gromov, Partial Differential Relations, Springer, 1986.

[4] D. McDuff and D. Salamon, Introduction to Symplectic Topology, Oxford University Press, 1995.

[5] C.H. Taubes, The Seiberg-Witten and Gromov Invariants, Math. Res. Lett. 2 (1995), 221-238.

[6] E. Witten, Monopoles and 4-manifolds, Math. Res. Lett. 1 (1994), 769-796.

[7] Y. Yomdin, The Geometry of Critical and Near-critical Values of Differentiable Mappings, Math. Annalen 264 (1983), 495-515.

Centre de Mathématiques, Ecole Polytechnique, 91128 Palaiseau, France

E-mail address: auroux@math.polytechnique.fr 